\title{
Influence of ring size on the reduction of vinylogous urethanes. Applications to the synthesis of lupinine and epilupinine
}

\author{
Joseph P. Michael,* Charles B. de Koning, Claudia San Fat, and Gillian L. Nattrass \\ Molecular Sciences Institute, School of Chemistry, University of the Witwatersrand, P.O. Wits, \\ 2050 South Africa \\ E-mail:jmichael@aurum.wits.ac.za
}

\begin{abstract}
Dedicated with respect and affection to Professor Jimmy Bull on his retirement from the University of Cape Town
\end{abstract}

\begin{abstract}
Short syntheses of the quinolizidine alkaloids lupinine and epilupinine via alkyl 3-(2-thioxo-1piperdinyl)propanoates and the vinylogous urethanes prepared from them are reported. Alkyl piperidin-2-ylideneacetates were found to undergo reduction of the $\mathrm{C}=\mathrm{C}$ bond with lithium aluminium hydride more readily than their pyrrolidin-2-ylideneacetate analogues, a finding that is in line with reports of the relative reactivities of double bonds exocyclic to five- and sixmembered rings.
\end{abstract}

Keywords: Enaminones, epilupinine, lupinine, quinolizidine alkaloids, thiolactams, vinylogous urethanes

\section{Introduction}

The general approach to alkaloid and antibiotic synthesis explored in these laboratories over a number of years is based on the use of $\beta$-acylated enamines (enaminones) and related compounds as scaffolds upon which to build the more complex architectures found in a representative range of alkaloidal families. ${ }^{1}$ The focus of our investigations has hitherto largely been on using 2-(acylmethylidene)pyrrolidines derived from pyrrolidine-2-thiones as versatile intermediates en route to target systems containing isolated or fused pyrrolidine rings, ${ }^{2}$ for example, the 1-azabicyclo[4.3.0]nonane (indolizidine) nucleus. ${ }^{3}$ Complementary explorations of piperidine-based precursors for the synthesis of the homologous 1-azabicyclo[4.4.0]decane (quinolizidine) ring system are under-represented in our work. ${ }^{4}$ However, our recently reported observations of intriguing discrepancies in the behavior of five-membered and six-membered thiolactam precursors ${ }^{5}$ have prompted us to examine the reactions of the latter class of 
compounds and their 2-alkylidene derivatives more closely, and to pay more attention to their use in the synthesis of quinolizidine alkaloids. Some relevant results are reported in this article.

The quinolizidine ring system is well represented among alkaloids isolated from both plant and animal sources, ${ }^{6}$ and novel strategies for the stereoselective synthesis of compounds containing this important structural motif continue to receive considerable attention. ${ }^{7}$ The diastereomeric alkaloids lupinine 1 and epilupinine $\mathbf{2}$ (numbering scheme shown), the simplest members of the large group of alkaloidal metabolites of the Leguminosae (Fabaceae), ${ }^{8}$ are exceptionally popular targets for synthesis, and are frequently used to exemplify emerging methodologies. ${ }^{9}$ In fact, an early communication from these laboratories disclosed a simple synthesis of $( \pm$ )-lupinine rac-1 from the piperidine-2-thione $\mathbf{3}$ via the enaminone intermediates 4-6 (vinylogous urethanes) (Scheme 1). ${ }^{10}$ However, full experimental details were never reported, the relatively unsophisticated spectroscopic techniques available at the time made the characterisation of compounds somewhat tentative, and the apparently stereoselective conversion of 6 into 1, although precedented, ${ }^{11}$ was not fully probed. We thus chose to re-investigate aspects of this synthesis, using the route as a framework within which to contextualise a further exploration of behavioral differences between five-and six-membered ring intermediates.

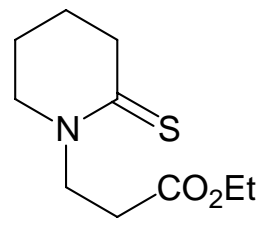

3

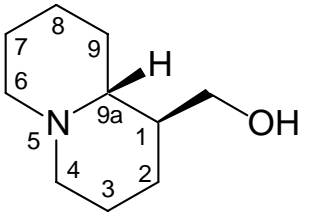

2 Epilupinine

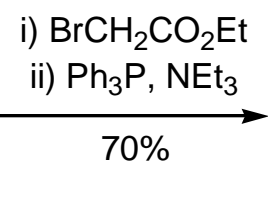

i) $\mathrm{BrCH}_{2} \mathrm{CO}_{2} \mathrm{Et}$

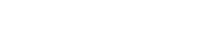

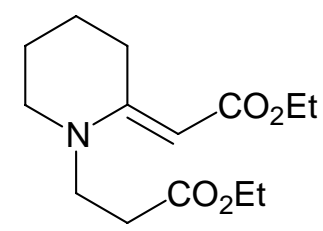

4

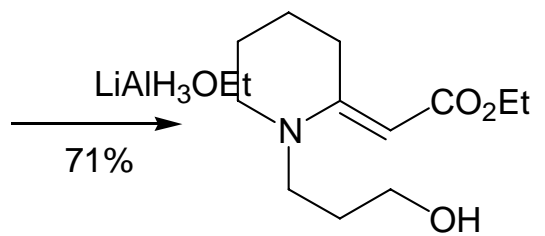

5

$\mathrm{NaH}, p-\mathrm{TsCl}$

ii) $\mathrm{MeCN}, \Delta$

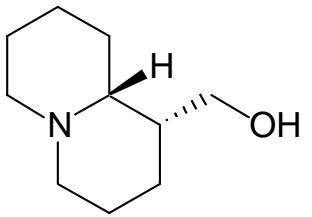

1 Lupinine

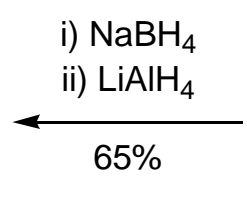

i) $\mathrm{NaBH}_{4}$

$65 \%$

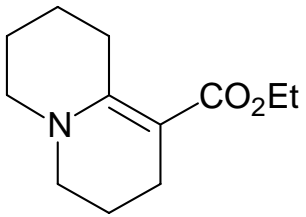

6

\section{Scheme 1}

\section{Results and Discussion}

The vinylogous urethanes on which this study is based were prepared in two steps from pyrrolidine-2-thione $^{12} \mathbf{7 a}$ or piperidine-2-thione ${ }^{12} \mathbf{7 b}$, conjugate addition of which to esters of acrylic acid was readily achieved by stirring the reactants together in dry tetrahydrofuran at 
$40{ }^{\circ} \mathrm{C}$ in the presence of a catalytic quantity of sodium hydride ${ }^{13}$ or sodium hydroxide (Scheme 2). The $N$-alkyl products 3 and $\mathbf{8 a}-\mathbf{c}$ were isolated in yields of $92-99 \%$. Conversion of these thiolactams into the vinylogous urethanes 4 and 9a-c was accomplished in 75-85\% overall yields by alkylation on sulfur with ethyl bromoacetate followed by Eschenmoser sulfide contraction ${ }^{14}$ in the presence of triphenylphosphine and triethylamine. The $(E)$-geometry of the double bond in these products was inferred from the chemical shift of the hydrogen atoms at C-3 in the ring $\left(\delta c a\right.$. 3.1), the downfield shift of about $0.6 \mathrm{ppm}$ relative to (Z)-analogues ${ }^{14 \mathrm{a}}$ arising from the anisotropic deshielding effect of the carbonyl group.

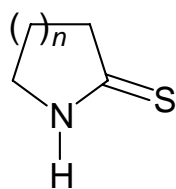

$7 a n=1$

$7 b \quad n=2$

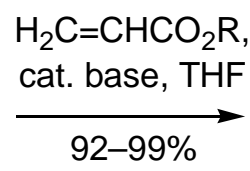

$92-99 \%$

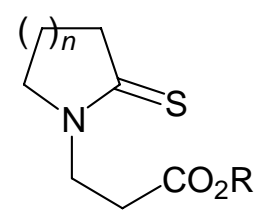

$3 n=2 ; \mathrm{R}=\mathrm{Et}$

$8 \mathbf{a} n=1 ; \mathrm{R}=\mathrm{Et}$

8b $n=1 ; \mathrm{R}=\mathrm{Bu}^{t}$

8c $n=2 ; \mathrm{R}=\mathrm{Bu}^{t}$ i) $\mathrm{BrCH}_{2} \mathrm{CO}_{2} \mathrm{Et}$

ii) $\mathrm{Ph}_{3} \mathrm{P}, \mathrm{NEt}_{3}$

$75-85 \%$

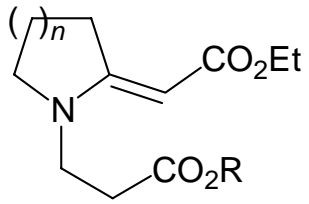

$4 n=2 ; \mathrm{R}=\mathrm{Et}$

9a $n=1 ; \mathrm{R}=\mathrm{Et}$

9b $n=1 ; \mathrm{R}=\mathrm{Bu}^{t}$

9c $n=2 ; \mathrm{R}=\mathrm{Bu}^{t}$

\section{Scheme 2}

A critical feature of the previously reported synthesis of lupinine shown in Scheme 1 was the chemoselective reduction of the saturated ester group of intermediate 4 while leaving the vinylogous urethane untouched. Our prior experience with pyrrolidine-based vinylogous urethanes led us to believe that the enaminone system in such compounds was robust enough to resist reduction with lithium aluminium hydride. ${ }^{1,3 \mathrm{~b}}$ Indeed, treatment of the pyrrolidinylidene compounds 9a and $9 \mathbf{b}$ with lithium aluminium hydride (1.1 equiv.) in tetrahydrofuran at room temperature was complete within $2 \mathrm{~h}$, affording the same saturated alcohol $\mathbf{1 0}$ in yields of 73\% and $79 \%$, respectively; as expected, the vinylogous urethane was unaffected. However, it was disconcerting to discover that the piperidinylidene analogues $\mathbf{4}$ and $9 \mathbf{c}$ were substantially less inert under the same conditions. The former, for instance, gave only a 3\% yield of the expected product 5, while the major product $\mathbf{1 1}(65 \%)$ resulted from reduction of both the saturated ester and the double bond of the vinylogous urethane. Even at a lower temperature $\left(0{ }^{\circ} \mathrm{C}\right)$ and with a shorter reaction time (45 min), the yield of 5 was only 66\%, while the over-reduced product $\mathbf{1 1}$ was still significant (26\%). Interestingly, in the original lupinine synthesis reported from this laboratory ${ }^{10}$ (Scheme 1), the reactivity of the reductant was tempered by the addition of an equivalent of ethanol ${ }^{15}$ to give a $71 \%$ yield of $\mathbf{5}$. Over-reduction was not mentioned, or, more probably, was not recognised. In the present work, attempts to moderate the reaction in other ways, e.g. by using Superhydride, ${ }^{\circledR}$ lithium pyrrolidineborohydride ${ }^{16}$ or sodium borohydride in combination with lithium iodide, ${ }^{17}$ failed to reduce either functional group. However, we were able to minimise over-reduction with lithium aluminium hydride by changing the solvent to a 
mixture of toluene and diethyl ether. With a 2:1 ratio of these solvents, reduction of 4 at $0{ }^{\circ} \mathrm{C}$ for 45 min afforded 5 (58\%) and $\mathbf{1 1}$ (27\%); with a 4:1 ratio of solvents, the isolated yields were $62 \%$ and $13 \%$, respectively.

The difference in reactivity of the exocyclic $\mathrm{C}=\mathrm{C}$ bond in the five- and six-membered vinylogous urethanes $\mathbf{4}$ and $\mathbf{9 a}-\mathbf{c}$ is in line with Brown's observations about the stability and reactivity of double bonds that are exocyclic or endocyclic to rings of varying size. ${ }^{18}$ Brown's carefully worded generalisation included the following statement: "Reactions which involve the loss of an exo double bond will be favored in the 6-ring as compared to the corresponding 5-ring derivative". Thus the greater lability of the piperidinylidene vinylogous urethanes towards conjugate reduction, though troublesome for our purposes, should perhaps not have surprised us. However, other factors may well make even this feature of enaminone reactivity unpredictable. For example, the vinylogous cyanamide 12a, the reduction of which to the alcohol $\mathbf{1 3}$ proceeds in $74 \%$ yield, ${ }^{4}$ is clearly far less susceptible to reduction of the $\mathrm{C}=\mathrm{C}$ bond than the corresponding vinylogous urethane. To confirm this observation and complete our series of comparisons, we prepared the tert-butyl ester analogue 12b in $75 \%$ yield by sulfide contraction between the piperidine-2-thione 8c and bromoacetonitrile. Reduction of the ester group with lithium aluminium hydride in THF at room temperature, although affording only a $60 \%$ yield of $\mathbf{1 3}$, gave no observable formation of the over-reduced product.

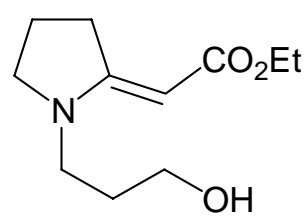

10

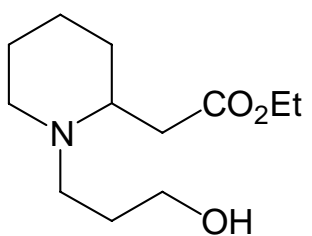

11

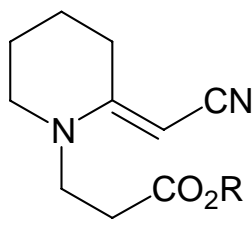

12a $\mathrm{R}=\mathrm{Et}$

12b $\mathrm{R}=\mathrm{Bu}^{t}$

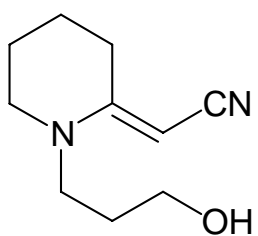

13

As originally performed, the cyclisation of alcohol $\mathbf{5}$ to give the quinolizidine ring system $\mathbf{6}$ (Scheme 1) entailed activation of the leaving group by initial conversion into a toluenesulfonate. We have subsequently found that this is not an ideal approach; since mixtures of toluenesulfonate and chloride products are formed, the latter in general not being sufficiently reactive to give efficient ring closure. Our preferred mode of activation is now via an alkyl iodide, which is formed in situ and immediately cyclised under appropriate conditions. In the present investigation, the alcohol $\mathbf{5}$ was treated with iodine, triphenylphosphine and imidazole, according to the method developed by Garegg and Samuelson ${ }^{19}$ for preparing alkyl iodides from alcohols. When the reactants were heated in a 2:1 mixture of toluene and acetonitrile, the intermediate iodide cyclised spontaneously to give the 3,4,6,7,8,9-hexahydro-2H-quinolizine 6 in $74 \%$ yield. Although other workers have prepared compound 6 by different routes, ${ }^{11,20,21}$ characterisation has hitherto been sketchy and ${ }^{13} \mathrm{C}$ NMR data have not previously been reported.

The selective conversion of $\mathbf{6}$ into the target quinolizidine alkaloids $\mathbf{1}$ and $\mathbf{2}$ demands that the reduction of the $\mathrm{C}=\mathrm{C}$ bond proceed with reliable stereocontrol. Goldberg and Ragade reported that 
stereocontrolled reduction of $\mathbf{6}$ with sodium borodeuteride gave the 9a-deuterated isotopomer of $( \pm$ )-ethyl lupinoate $\mathbf{1 4}$ exclusively, a somewhat surprising result that was rationalised in terms of conformational effects and the preferred trajectory of hydride approach. ${ }^{11}$ Lhommet and coworkers prepared 14 by catalytic hydrogenation of $\mathbf{6}$ over Raney nickel under harsh conditions $\left(150 \mathrm{~atm} ., 100{ }^{\circ} \mathrm{C}\right) .{ }^{21 b}$ Significantly, at a reaction temperature of $200^{\circ} \mathrm{C}$, they found that the sole product was ( \pm )-ethyl epilupinoate 15 , in which the ester group occupies the thermodynamically favoured equatorial position. Furthermore, $\mathbf{1 4}$ could be epimerised to $\mathbf{1 5}$ merely upon heating at $200{ }^{\circ} \mathrm{C}$. Less drastically, Goldberg and Lipkin accomplished epimerisation in unspecified yield by heating the lupinate ester $\mathbf{1 4}$ with sodium ethoxide in ethanol. ${ }^{20}$

We found that stereoselective cis-hydrogenation of the bicyclic vinylogous urethane $\mathbf{6}$ could be accomplished under far milder conditions than those employed by Lhommet's group. Hydrogenation over Adams catalyst in absolute ethanol at a hydrogen pressure of 5 atm afforded ( \pm )-ethyl lupinoate $\mathbf{1 4}$ as the sole detectable isomer in a yield of 83\% (Scheme 3). Moreover, in our hands the base-catalysed epimerisation with a catalytic quantity of sodium ethoxide in boiling ethanol gave a quantitative yield of the epilupinoate ester 15. Although both isomers have been known for decades, reliable spectroscopic data are rare. Our spectroscopic data agreed well those of Lhommet ${ }^{21 b}$ and also Hua et al., ${ }^{22}$ who reported data for the enantiomerically pure compounds (-)-14 and (+)-15. In the IR spectra, Bohlmann bands ${ }^{23}$ in the region ca. 2750-2800 $\mathrm{cm}^{-1}$ indicated trans-fusion of the quinolizidine ring system.

Reduction of ( \pm )-14 and ( \pm )-15 to give ( \pm )-lupinine 1 and ( \pm )-epilupinine 2, respectively, was most successfully achieved by the dropwise addition of the precursors in dry diethyl ether to a suspension of lithium aluminium hydride in ether at $0{ }^{\circ} \mathrm{C}$, a "reverse addition" procedure recommended by Davies and Smyth. ${ }^{24}$ The isolated yields of the two alkaloids were $95 \%$ and $88 \%$, respectively. Spectroscopic data for these products, recorded in deuterated chloroform at $300 \mathrm{MHz}$, were in excellent agreement with those recorded at $400 \mathrm{MHz}$ by Hua et al. ${ }^{22}$ In addition, spectra recorded for lupinine in deuterated benzene accorded well with those reported at $600 \mathrm{MHz}$ by Rycroft and Robins. ${ }^{25}$

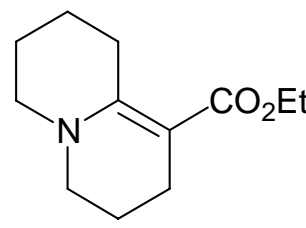

6
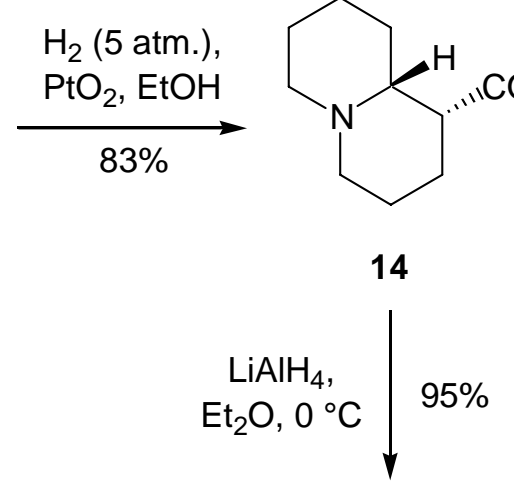

1 ( \pm )-Lupinine

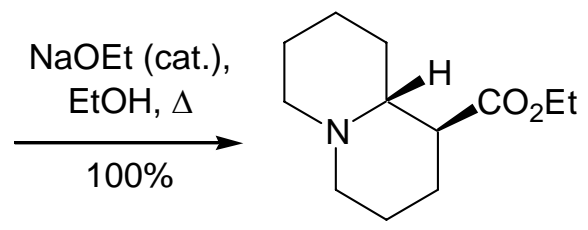

15

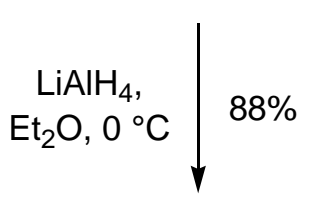

2 ( \pm )-Epilupinine

Scheme 3 
The methodology described in this article is conceptually and experimentally straightforward and the transformations are efficient. We are currently applying the principles elaborated above to the enantioselective synthesis of a suite of 1,4-disubstituted quinolizidine alkaloids recently isolated from the skins of poison-dart frogs. ${ }^{26}$

\section{Experimental Section}

General Procedures. All solvents used for reactions and chromatography were distilled before use. Tetrahydrofuran (THF) and diethyl ether were distilled from $\mathrm{Na}$ /benzophenone, dichloromethane, acetonitrile and triethylamine from $\mathrm{CaH}_{2}$, and benzene and toluene from Na. Commercially available chemicals were used as received. Melting points, recorded on a Reichert hot-stage microscope apparatus, are uncorrected. TLC was performed on aluminium-backed Alugram Sil G/UV 254 plates pre-coated with $0.25 \mathrm{~mm}$ silica gel 60 . Column chromatography was carried out on silica gel 60, particle size $0.063-0.200 \mathrm{~mm}$ (conventional columns) or Whatman Partisil Prep 40, particle size 0.040-0.063 mm (flash columns). FTIR spectra were recorded on a Bruker Vector 22 spectrometer. NMR spectra were recorded on a Bruker AC-200 (200.13 MHz for ${ }^{1} \mathrm{H}, 50.32 \mathrm{MHz}$ for ${ }^{13} \mathrm{C}$ ), Bruker AVANCE 300 (300.132 MHz for ${ }^{1} \mathrm{H}, 75.473 \mathrm{MHz}$ for ${ }^{13} \mathrm{C}$ ) or Bruker DRX 400 (400.132 MHz for ${ }^{1} \mathrm{H}, 100.625 \mathrm{MHz}$ for ${ }^{13} \mathrm{C}$ ) spectrometers. $\mathrm{CDCl}_{3}$ was used as solvent and TMS as internal standard. DEPT and CH-correlated spectra were routinely used for assignment of signals. $J$ values are given in Hz. High-resolution mass spectra were recorded at $70 \mathrm{eV}$ on a VG 70 SEQ mass spectrometer with a MASPEC II data system.

\section{General procedure for the $\mathrm{N}$-alkylation of thiolactams with acrylate esters}

To a stirred solution of pyrrolidine-2-thione ${ }^{12} \mathbf{7 a}$ or piperidine-2-thione ${ }^{12} \mathbf{7 b}$ (5-45 mmol scale) in dry THF (ca. $1.75 \mathrm{~cm}^{3}$ per mmol of solute) was added a catalytic amount of $\mathrm{NaH}(60 \%$ dispersion in oil, ca. 0.05 equiv.) or $\mathrm{NaOH}$, followed by the dropwise addition of the acrylate ester (1.2 equiv.). The mixture was stirred at $40{ }^{\circ} \mathrm{C}$ for $16 \mathrm{~h}$ before evaporating the solvent in vacuo. The resulting yellow residue was dissolved in $\mathrm{Et}_{2} \mathrm{O}$, and the resulting solution was washed with $\mathrm{H}_{2} \mathrm{O}$ and saturated aqueous $\mathrm{NaCl}$ solution. The ethereal phase was dried $\left(\mathrm{MgSO}_{4}\right)$, filtered and evaporated in vacuo to yield a yellow oil, which was purified by column chromatography on silica gel using EtOAc-hexane (1:9 to 1:1) as eluent to give the following products:

Ethyl 3-(2-thioxo-1-piperidinyl)propanoate (3). (6.89 g, 92\%), from piperidine-2-thione 7b (4.00 g, $34.72 \mathrm{mmol}$ ), dry THF (60 $\left.\mathrm{cm}^{3}\right)$, NaH dispersion (69 mg, $1.74 \mathrm{mmol}$ ) and ethyl acrylate $\left(4.49 \mathrm{~cm}^{3}, 4.19 \mathrm{~g}, 41.67 \mathrm{mmol}\right.$ ); pale yellow oil, $\mathrm{R}_{f} 0.56$ (EtOAc-hexane 1:1); $v_{\max }(\mathrm{film}) / \mathrm{cm}^{-1}$ 2947 (m, br), 1732 (s, C=O), 1520 (s), 1353 (m, C=S), 1184 (s), 1159 (s), 1098 (m) and 1051 (m); $\delta_{\mathrm{H}}\left(400 \mathrm{MHz} ; \mathrm{CDCl}_{3}\right) 4.19$ (2H, t, $J$ 6.9, chain $\left.\mathrm{NCH}_{2}\right), 4.14$ (2H, q, $J$ 7.1, $\left.\mathrm{OCH}_{2} \mathrm{CH}_{3}\right), 3.53$ $\left(2 \mathrm{H}, \mathrm{t}, J\right.$ 6.1, ring $\left.\mathrm{NCH}_{2}\right), 2.97\left(2 \mathrm{H}, \mathrm{t}, J\right.$ 6.5, $\left.\mathrm{CH}_{2} \mathrm{C}=\mathrm{S}\right), 2.85\left(2 \mathrm{H}, \mathrm{t}, J\right.$ 7.0, $\left.\mathrm{CH}_{2} \mathrm{CO}_{2} \mathrm{Et}\right), 1.89(2 \mathrm{H}$, quintet, $J$ 6.2, ring 5-H), 1.72 (2H, quintet, $J$ 6.4, ring 4-H) and 1.27 (3H, t, $J$ 7.1, $\mathrm{OCH}_{2} \mathrm{CH}_{3}$ ); $\delta_{\mathrm{C}}$ 
(100 MHz; $\left.\mathrm{CDCl}_{3}\right) 199.9(\mathrm{C}=\mathrm{S}), 171.6(\mathrm{C}=\mathrm{O}), 60.6\left(\mathrm{OCH}_{2} \mathrm{CH}_{3}\right), 51.8$ (ring $\mathrm{NCH}_{2}$ ), 50.7 (chain $\left.\mathrm{NCH}_{2}\right)$, $41.6\left(\mathrm{CH}_{2} \mathrm{C}=\mathrm{S}\right), 30.7\left(\mathrm{CH}_{2} \mathrm{CO}_{2} \mathrm{Et}\right), 22.8$ (ring $\left.\mathrm{C}-5\right)$, 20.3 (ring $\left.\mathrm{C}-4\right)$ and 14.0 $\left(\mathrm{OCH}_{2} \mathrm{CH}_{3}\right)$. The spectroscopic data agree with those published previously. ${ }^{4}$

Ethyl 3-(2-thioxo-1-pyrrolidinyl)propanoate (8a). (0.85g, 92\%), from pyrrolidine-2-thione 7a (0.463 g, $4.58 \mathrm{mmol})$, dry THF $\left(10 \mathrm{~cm}^{3}\right), \mathrm{NaOH}(<10 \mathrm{mg})$ and ethyl acrylate $\left(0.55 \mathrm{~cm}^{3}, 5.95\right.$ mmol); yellow oil, $\mathrm{R}_{f} 0.54$ (EtOAc-hexane 1:1); $v_{\max }\left(\right.$ thin film)/ $\mathrm{cm}^{-1} 2979$ (m), 1732 (s, C=O), $1511(\mathrm{~m}), 1379(\mathrm{~m}), 1320(\mathrm{~m})$ and $1188(\mathrm{~m}) ; \delta_{\mathrm{H}}\left(400 \mathrm{MHz} ; \mathrm{CDCl}_{3}\right) 4.15(2 \mathrm{H}, \mathrm{q}, J$ 7.2, $\left.\mathrm{OCH}_{2} \mathrm{CH}_{3}\right), 4.01\left(2 \mathrm{H}, \mathrm{t}, J\right.$ 6.8, chain $\left.\mathrm{NCH}_{2}\right), 3.82\left(2 \mathrm{H}, \mathrm{t}, J\right.$ 7.3, ring $\left.\mathrm{NCH}_{2}\right), 3.01(2 \mathrm{H}, \mathrm{t}, J$ 7.9, $\mathrm{CH}_{2} \mathrm{C}=\mathrm{S}$ ), $2.77\left(2 \mathrm{H}, \mathrm{t}, J\right.$ 6.8, $\mathrm{CH}_{2} \mathrm{CO}_{2} \mathrm{Et}$ ), $2.08(2 \mathrm{H}$, quintet, $J$ 7.6, ring 4-H), 1.27 (3H, t, $J$ 7.2, $\left.\mathrm{OCH}_{2} \mathrm{CH}_{3}\right) ; \delta_{\mathrm{C}}\left(50 \mathrm{MHz} ; \mathrm{CDCl}_{3}\right) 201.4(\mathrm{C}=\mathrm{S}), 171.2(\mathrm{C}=\mathrm{O}), 60.6\left(\mathrm{OCH}_{2} \mathrm{CH}_{3}\right), 55.6$ (ring $\left.\mathrm{NCH}_{2}\right)$, 44.7 (chain $\left.\mathrm{NCH}_{2}\right), 43.5\left(\mathrm{CH}_{2} \mathrm{C}=\mathrm{S}\right), 30.8\left(\mathrm{CH}_{2} \mathrm{CO}_{2} \mathrm{Et}\right), 19.7$ (ring C-4), 13.9 $\left(\mathrm{OCH}_{2} \mathrm{CH}_{3}\right) ; \mathrm{m} / \mathrm{z}$ (EI) 201 (31\%, M+), 172 (21), 156 (26), 129 (24), 128 (100), 126 (14), 114 (26), 112 (33), 111 (39), 110 (17), 87 (14), 85 (84). The compound has previously been reported, but without spectroscopic characterisation. ${ }^{27}$

tert-Butyl 3-(2-thioxo-1-pyrrolidinyl)propanoate (8b). (1.13 g, 99\%), from pyrrolidine-2thione $7 \mathbf{a}(0.58 \mathrm{~g}, 5.73 \mathrm{mmol})$, dry THF $\left(10 \mathrm{~cm}^{3}\right)$, NaH dispersion (11 mg, $\left.0.29 \mathrm{mmol}\right)$ and tertbutyl acrylate $\left(1.00 \mathrm{~cm}^{3}, 0.88 \mathrm{~g}, 6.88 \mathrm{mmol}\right.$ ); yellow oil, $\mathrm{R}_{f} 0.33$ (EtOAc-hexane 3:7); $v_{\max }$ (film)/cm 2978 (m), 1726 (s, C=O), 1509 (m), 1367 (m), 1326 (m), 1289 (m), 1254 (m), 1154 (s) and $1120(\mathrm{~m}) ; \delta_{\mathrm{H}}\left(400 \mathrm{MHz} ; \mathrm{CDCl}_{3}\right) 3.98\left(2 \mathrm{H}, \mathrm{t}, J\right.$ 6.8, chain $\left.\mathrm{NCH}_{2}\right), 3.80(2 \mathrm{H}, \mathrm{t}, J$ 7.3, ring $\mathrm{NCH}_{2}$ ), 3.00 (2H, t, $J$ 7.9, $\left.\mathrm{CH}_{2} \mathrm{C}=\mathrm{S}\right), 2.67\left(2 \mathrm{H}, \mathrm{t}, J 6.8, \mathrm{CH}_{2} \mathrm{CO}_{2} \mathrm{R}\right), 2.06$ (2H, quintet, $J$ 7.6, ring $\mathrm{H}-4)$ and 1.46 (s, 9H, $\left.\mathrm{CMe}_{3}\right) ; \delta_{\mathrm{C}}\left(50 \mathrm{MHz} ; \mathrm{CDCl}_{3}\right) 201.4(\mathrm{C}=\mathrm{S}), 170.7(\mathrm{C}=\mathrm{O}), 81.1\left(\mathrm{CMe}_{3}\right)$, 55.6 (ring $\left.\mathrm{NCH}_{2}\right), 44.8\left(\mathrm{CH}_{2} \mathrm{C}=\mathrm{S}\right), 43.8$ (chain $\left.\mathrm{NCH}_{2}\right), 32.3\left(\mathrm{CH}_{2} \mathrm{CO}_{2} \mathrm{R}\right), 27.9\left(\mathrm{CMe}_{3}\right)$ and 19.8 (ring C-4); m/z (EI) 229 (57\%, M ), 173 (34), 172 (38) 156 (42), 128 (100), 114 (10), 102 (14), 101 (6), 85 (17), 57 (14) (Found: $\mathrm{M}^{+}, 229.1140 . \mathrm{C}_{11} \mathrm{H}_{19} \mathrm{NO}_{2} \mathrm{~S}$ requires 229.1137. $\mathrm{M}^{+}-\mathrm{CO}_{2} \mathrm{Bu}^{t}$, 128.0530. $\mathrm{C}_{6} \mathrm{H}_{10} \mathrm{NS}$ requires 128.0534).

tert-Butyl 3-(2-thioxo-1-piperidinyl)propanoate (8c). (10.57 g, 99\%), from piperidine-2-thione $7 \mathbf{b}$ (5.00 g, $43.40 \mathrm{mmol})$, dry THF $\left(75 \mathrm{~cm}^{3}\right)$, NaH dispersion ( $\left.87 \mathrm{mg}, 2.17 \mathrm{mmol}\right)$ and tert-butyl acrylate $\left(7.60 \mathrm{~cm}^{3}, 6.71 \mathrm{~g}, 52.36 \mathrm{mmol}\right.$ ); pale yellow solid, m.p. $55-56{ }^{\circ} \mathrm{C}$ (from pentane); $\mathrm{R}_{f}$ 0.66 (EtOAc-hexane 1:1); $v_{\max }$ (film)/ $/ \mathrm{cm}^{-1} 2950$ (m), 1724 (s, C=O), 1521 (s), 1450 (s), 1360 $(\mathrm{m}, \mathrm{C}=\mathrm{S}), 1160(\mathrm{~m})$ and $966(\mathrm{~m}) ; \delta_{\mathrm{H}}\left(400 \mathrm{MHz} ; \mathrm{CDCl}_{3}\right) 4.16\left(2 \mathrm{H}, \mathrm{t}, J\right.$ 7.0, chain $\left.\mathrm{NCH}_{2}\right), 3.53$ $\left(2 \mathrm{H}, \mathrm{t}, J\right.$ 6.1, ring $\left.\mathrm{NCH}_{2}\right), 2.97\left(2 \mathrm{H}, \mathrm{t}, J 6.5, \mathrm{CH}_{2} \mathrm{C}=\mathrm{S}\right), 2.76\left(2 \mathrm{H}, \mathrm{t}, J 7.0, \mathrm{CH}_{2} \mathrm{CO}_{2} \mathrm{R}\right), 1.88(2 \mathrm{H}$, quintet, $J$ 6.4, ring 5-H), $1.72(2 \mathrm{H}$, quintet, $J$ 6.4, ring $4-\mathrm{H})$ and $1.45\left(9 \mathrm{H}, \mathrm{s}, \mathrm{CMe}_{3}\right)$; $\delta_{\mathrm{C}}(100$ $\left.\mathrm{MHz} ; \mathrm{CDCl}_{3}\right) 199.7(\mathrm{C}=\mathrm{S}), 170.9(\mathrm{C}=\mathrm{O}), 80.7\left(\mathrm{CMe}_{3}\right), 51.7$ (ring $\mathrm{NCH}_{2}$ ), 50.8 (chain $\mathrm{NCH}_{2}$ ), $41.6\left(\mathrm{CH}_{2} \mathrm{C}=\mathrm{S}\right), 31.8\left(\mathrm{CH}_{2} \mathrm{CO}_{2} \mathrm{R}\right), 27.9\left(\mathrm{CMe}_{3}\right), 22.8$ (ring $\left.\mathrm{C}-5\right)$ and 20.2 (ring $\left.\mathrm{C}-4\right) ; \mathrm{m} / \mathrm{z}(\mathrm{EI})$ 243 (33\%, M+), 187(21), 186 (100), 170 (34), 142 (44), 116 (15), 115 (21), 82 (23) (Found: C, 59.51; $\mathrm{H}, 8.84 ; \mathrm{N}, 5.76 . \mathrm{C}_{12} \mathrm{H}_{21} \mathrm{NO}_{2} \mathrm{~S}$ requires $\mathrm{C}$, 59.23; H, 8.70; N, 5.76\%).

\section{General procedure for the preparation of vinylogous urethanes from $\boldsymbol{N}$-alkylthiolactams}

Ethyl bromoacetate (1.1 equiv.) was added dropwise to a stirred solution of thiolactam $\mathbf{3}$ or $\mathbf{8 a - c}$ (2-20 mmol scale) in dry $\mathrm{CH}_{3} \mathrm{CN}$ (ca. $1.75 \mathrm{~cm}^{3}$ per mmol of solute). The resulting solution was 
stirred at room temperature for $16 \mathrm{~h}$, after which S-alkylation was complete. The volatiles were removed in vacuo (oil pump), and the residue was thoroughly dried under vacuum. This salt was then re-dissolved in dry $\mathrm{CH}_{3} \mathrm{CN}$ ( $c$. $1.75 \mathrm{~cm}^{3}$ per mmol of solute), after which triphenylphosphine (1.1 equiv.) and triethylamine (1.1 equiv.) were added to induce sulfur extrusion. The resulting solution was left to stir at ambient temperature for $16 \mathrm{~h}$ ( $2.25 \mathrm{~h}$ for $\mathbf{8 b}$ ). $\mathrm{H}_{2} \mathrm{O}\left(10-20 \mathrm{~cm}^{3}\right)$ was added, and the resulting solution was extracted repeatedly with $\mathrm{CH}_{2} \mathrm{Cl}_{2}$. The organic extracts were dried $\left(\mathrm{MgSO}_{4}\right)$, filtered and evaporated in vacuo. The crude product was purified by column chromatography on silica gel; $\mathrm{CH}_{2} \mathrm{Cl}_{2}$ or hexane-benzene (9:1) were used initially as eluents to remove triphenylphosphine and triphenylphosphine sulfides, after which the desired products were eluted with hexane-EtOAc mixtures (8:2 to 7:3). The following products were obtained:

Ethyl 3-[(2E)-2-(2-ethoxy-2-oxoethylidene)piperidinyl]propanoate (4). (4.63 g, 84\%), from ethyl 3-(2-thioxo-1-piperidinyl)propanoate 3 (4.00 g, $18.55 \mathrm{mmol}$ ), ethyl bromoacetate (2.27 $\left.\mathrm{cm}^{3}, 3.41 \mathrm{~g}, 24.43 \mathrm{mmol}\right)$, dry $\mathrm{CH}_{3} \mathrm{CN}\left(2 \times 35 \mathrm{~cm}^{3}\right), \mathrm{PPh}_{3}(5.36 \mathrm{~g}, 20.43 \mathrm{mmol})$ and $\mathrm{NEt}_{3}(2.85$ $\mathrm{cm}^{3}, 2.07 \mathrm{~g}, 20.43 \mathrm{mmol}$ ); yellow oil, $\mathrm{R}_{f} 0.69$ (EtOAc-hexane 1:1); $v_{\max }(\mathrm{film}) / \mathrm{cm}^{-1} 2980(\mathrm{w})$, 2943 (w), 1733 (m, C=O), 1681 (m, C=O), 1566 (s, C=C), 1187 (m), 1134 (s), 1096 (m), 1050 (m) and $790(\mathrm{w}) ; \delta_{\mathrm{H}}\left(400 \mathrm{MHz} ; \mathrm{CDCl}_{3}\right) 4.55(1 \mathrm{H}, \mathrm{s},=\mathrm{CH}), 4.15\left(2 \mathrm{H}, \mathrm{q}, J\right.$ 7.1, $\left.\mathrm{OCH}_{2} \mathrm{CH}_{3}\right), 4.06$ (2H, q, $J$ 7.1, $\mathrm{OCH}_{2} \mathrm{CH}_{3}$ ), 3.49 (2H, t, $J$ 7.1, chain $\mathrm{NCH}_{2}$ ), 3.27 (2H, t, $J$ 6.1, ring $\mathrm{NCH}_{2}$ ), 3.09 (2H, t, $J$ 6.6, $\left.\mathrm{CH}_{2} \mathrm{C}=\right), 2.62\left(2 \mathrm{H}, \mathrm{t}, J\right.$ 7.2, $\left.\mathrm{CH}_{2} \mathrm{CO}_{2} \mathrm{R}\right), 1.76$ (2H, quintet, $J$ 6.2, ring 4-H), 1.62 (2H, quintet, $J 6.4$, ring 5-H) and 1.27 and $1.24\left(6 \mathrm{H}\right.$, overlapping t, $\left.J 7.1,2 \times \mathrm{OCH}_{2} \mathrm{CH}_{3}\right) ; \delta_{\mathrm{C}}$ (100 MHz; $\left.\mathrm{CDCl}_{3}\right) 171.5(\mathrm{C}=\mathrm{O}$, saturated ester), $168.6(\mathrm{C}=\mathrm{O}$, unsaturated ester), 161.3 $(\mathrm{NC}=\mathrm{CH}), 82.0(\mathrm{NC}=\mathrm{CH}), 60.5$ and $57.9\left(2 \times \mathrm{OCH}_{2} \mathrm{CH}_{3}\right), 49.9\left(\right.$ ring $\left.\mathrm{NCH}_{2}\right), 47.4$ (chain $\left.\mathrm{NCH}_{2}\right)$, $30.3\left(\mathrm{CH}_{2} \mathrm{CO}_{2} \mathrm{R}\right), 26.2\left(\mathrm{CH}_{2} \mathrm{C}=\right)$, 23.2 (ring $\left.\mathrm{C}-4\right), 19.3$ (ring $\left.\mathrm{C}-5\right), 14.5$ and $14.0(2 \times$ $\mathrm{OCH}_{2} \mathrm{CH}_{3}$ ); $\mathrm{m} / \mathrm{z}$ (EI) 269 (40\%, M+), 224 (62), 196 (100), 182 (50), 169 (31), 154 (7), 149 (15), 124 (20), 97 (48) (Found: $\mathrm{M}^{+}$, 269.1626. $\mathrm{C}_{14} \mathrm{H}_{23} \mathrm{NO}_{4}$ requires 269.1627).

Ethyl 3-[(2E)-2-(2-ethoxy-2-oxoethylidene)pyrrolidinyl]propanoate (9a). (0.61 g, 83\%), from ethyl 3-(2-thioxo-1-pyrrolidinyl)propanoate 8a $(0.58 \mathrm{~g}, 2.88 \mathrm{mmol})$, ethyl bromoacetate $\left(0.35 \mathrm{~cm}^{3}, 0.53 \mathrm{~g}, 3.17 \mathrm{mmol}\right)$, dry $\mathrm{CH}_{3} \mathrm{CN}\left(2 \times 5 \mathrm{~cm}^{3}\right), \mathrm{PPh}_{3}(0.83 \mathrm{~g}, 3.17 \mathrm{mmol})$ and $\mathrm{NEt}_{3}(0.44$ $\mathrm{cm}^{3}, 0.32 \mathrm{~g}, 3.17 \mathrm{mmol}$ ); waxy solid, m.p. $31-33{ }^{\circ} \mathrm{C} ; \mathrm{R}_{f} 0.54$ (EtOAc-hexane 1:1); $v_{\max }$ (film)/cm $\mathrm{cm}^{-1} 2980$ (m), 1734 (s, C=O), 1638 (s, C=O), 1595 (s, C=C), 1377 (m), 1301 (m), 1250 (m), 1134 (s, br), $1052(\mathrm{~m})$ and $786(\mathrm{~m}) ; \delta_{\mathrm{H}}\left(200 \mathrm{MHz}^{\left.-\mathrm{CDCl}_{3}\right)} 4.53(1 \mathrm{H}, \mathrm{s},=\mathrm{CH}), 4.15(2 \mathrm{H}, \mathrm{q}, J\right.$ 7.1, $\left.\mathrm{OCH}_{2} \mathrm{CH}_{3}\right), 4.09$ (2H, q, $J$ 7.1, $\left.\mathrm{OCH}_{2} \mathrm{CH}_{3}\right), 3.50\left(2 \mathrm{H}, \mathrm{t}, J\right.$ 9.0, chain $\left.\mathrm{NCH}_{2}\right), 3.41(2 \mathrm{H}, \mathrm{t}, J$ 7.0, ring $\left.\mathrm{NCH}_{2}\right), 3.13\left(2 \mathrm{H}\right.$, td, $J 7.8$ and $\left.1.1, \mathrm{CH}_{2} \mathrm{C}=\right), 2.58\left(2 \mathrm{H}, \mathrm{t}, J 7.0, \mathrm{CH}_{2} \mathrm{CO}_{2} \mathrm{R}\right), 1.93(2 \mathrm{H}$, quintet, $J$ 7.4, ring $\mathrm{H}-4)$ and 1.27 and $1.25\left(6 \mathrm{H}\right.$, overlapping t, $\left.J 7.1,2 \times \mathrm{OCH}_{2} \mathrm{CH}_{3}\right) ; \delta_{\mathrm{C}}(50$ $\left.\mathrm{MHz} ; \mathrm{CDCl}_{3}\right) 171.4(\mathrm{C}=\mathrm{O}$, saturated ester), 169.1 ( $\mathrm{C}=\mathrm{O}$, unsaturated ester), $164.3(\mathrm{NC}=\mathrm{CH}$ ), $78.1\left(\mathrm{NC}=\mathrm{CH}\right.$ ), 60.7 and $58.1\left(2 \times \mathrm{OCH}_{2} \mathrm{CH}_{3}\right), 52.7$ (ring $\left.\mathrm{NCH}_{2}\right), 41.8$ (chain $\left.\mathrm{NCH}_{2}\right), 32.4$ $\left(\mathrm{CH}_{2} \mathrm{C}=\right), 30.8\left(\mathrm{CH}_{2} \mathrm{CO}_{2} \mathrm{R}\right), 21.0$ (ring $\left.\mathrm{C}-4\right), 14.6$ and $14.0\left(2 \times \mathrm{OCH}_{2} \mathrm{CH}_{3}\right) ; \mathrm{m} / \mathrm{z} 255\left(11 \%, \mathrm{M}^{+}\right)$, 210 (17), 185 (39), 182 (17), 140 (31), 128 (20), 114 (20), 112 (42), 111 (55), 110 (20), 101 (28), 98 (100), 94 (18), 85 (17) (Found: C, 61.18; H, 8.23; N, 5.45. $\mathrm{C}_{13} \mathrm{H}_{21} \mathrm{NO}_{4}$ requires C, 61.16; $\mathrm{H}$, 8.29; N, 5.49\%). The compound has previously been reported, but without spectroscopic data. ${ }^{27}$ 
tert-Butyl 3-[(2E)-2-(2-ethoxy-2-oxoethylidene)pyrrolidinyl]propanoate (9b). (0.44 g, 75\%), from tert-butyl 3-(2-thioxo-1-pyrrolidinyl)propanoate $\mathbf{8 b}(0.53 \mathrm{~g}, 2.31 \mathrm{mmol})$, ethyl bromoacetate $\left(0.28 \mathrm{~cm}^{3}, 0.42 \mathrm{~g}, 2.54 \mathrm{mmol}\right)$, dry $\mathrm{CH}_{3} \mathrm{CN}\left(2 \times 4 \mathrm{~cm}^{3}\right), \mathrm{PPh}_{3}(0.67 \mathrm{~g}, 2.54 \mathrm{mmol})$ and $\mathrm{NEt}_{3}\left(0.35 \mathrm{~cm}^{3}, 0.26 \mathrm{~g}, 2.54 \mathrm{mmol}\right.$ ); yellow oil, $\mathrm{R}_{f} 0.69$ (EtOAc-hexane 1:1); $v_{\max }$ (film)/cm ${ }^{-1} 2978$ (m), 2935 (m), 1728 (s, C=O), 1685 (s, C=O), 1595 (s, C=C), 1368 (m), 1301 (m), $1249(\mathrm{~m}), 1133(\mathrm{~s})$ and $1051(\mathrm{~m}) ; \delta_{\mathrm{H}}\left(400 \mathrm{MHz} ; \mathrm{CDCl}_{3}\right) 4.53(1 \mathrm{H}, \mathrm{s},=\mathrm{CH}), 4.09$ (2H, q, $J$ 7.1, $\mathrm{OCH}_{2} \mathrm{CH}_{3}$ ), 3.45 (2H, t, $J$ 7.0, chain $\mathrm{NCH}_{2}$ ), 3.39 (2H, t, $J$ 7.1, ring $\mathrm{NCH}_{2}$ ), 3.13 (2H, t, $J$ 7.8, $\mathrm{CH}_{2} \mathrm{C}=$ ), 2.49 (2H, t, J 7.0, $\left.\mathrm{CH}_{2} \mathrm{CO}_{2} \mathrm{R}\right), 1.92$ (2H, quintet, $J$ 7.4, ring 4-H), $1.45(9 \mathrm{H}, \mathrm{s}$, $\mathrm{CMe}_{3}$ ), 1.25 (3H, t, $J$ 7.2, $\left.\mathrm{OCH}_{2} \mathrm{CH}_{3}\right) ; \delta_{\mathrm{C}}\left(100 \mathrm{MHz} ; \mathrm{CDCl}_{3}\right) 170.7$ ( $C=\mathrm{O}$, saturated ester), 169.3 ( $C=\mathrm{O}$, unsaturated ester), $164.5(\mathrm{NC}=\mathrm{CH}), 81.0\left(\mathrm{CMe}_{3}\right), 78.1(\mathrm{NC}=\mathrm{CH}), 58.2\left(\mathrm{OCH}_{2} \mathrm{CH}_{3}\right), 52.7$ (ring $\left.\mathrm{NCH}_{2}\right)$, 42.0 (chain $\left.\mathrm{NCH}_{2}\right), 32.5\left(\mathrm{CH}_{2} \mathrm{C}=\right)$ ), $32.2\left(\mathrm{CH}_{2} \mathrm{CO}_{2} \mathrm{R}\right), 28.0\left(\mathrm{CMe}_{3}\right), 21.1$ (ring C-4), $14.7\left(\mathrm{OCH}_{2} \mathrm{CH}_{3}\right) ; \mathrm{m} / \mathrm{z}$ (EI) 283 (53\%, M ${ }^{+}$), 238 (36), 227 (17), 226 (10), 210 (43), 183 (18), 182 (100), 168 (35), 155 (62), 154 (38), 111 (11), 110 (17), 108 (19), 57 (14) (Found: M $^{+}, 283.1788$; $\mathrm{C}_{15} \mathrm{H}_{25} \mathrm{NO}_{4}$ requires 283.1784)

tert-Butyl 3-[(2E)-2-(2-ethoxy-2-oxoethylidene)piperidinyl]propanoate (9c). (0.94 g, 85\%), from tert-butyl 3-(2-thioxo-1-piperidinyl)propanoate $8 \mathrm{8c}(900 \mathrm{mg}, 3.70 \mathrm{mmol})$, ethyl bromoacetate $\left(0.45 \mathrm{~cm}^{3}\right.$, 0.68.g, $\left.4.07 \mathrm{mmol}\right)$, dry $\mathrm{CH}_{3} \mathrm{CN}\left(2 \times 7 \mathrm{~cm}^{3}\right), \mathrm{PPh}_{3}(1.07 \mathrm{~g}, 4.07 \mathrm{mmol})$ and $\mathrm{NEt}_{3}\left(0.58 \mathrm{~cm}^{3}, 0.41 \mathrm{~g}, 4.07 \mathrm{mmol}\right.$ ); pale yellow oil, $\left(\mathrm{R}_{f} 0.37\right.$ (EtOAc-hexane 1:1); $v_{\max }$ (film)/cm ${ }^{-1} 2978$ (m), 2938 (m), 1728 (s, C=O), 1683 (s, C=O), 1568 (s, C=C), 1368 (m), 1332 (m), $1259(\mathrm{~m}), 1135$ (s, br), $1096(\mathrm{~m})$ and $1051(\mathrm{~m}) ; \delta_{\mathrm{H}}\left(200 \mathrm{MHz} ; \mathrm{CDCl}_{3}\right) 4.55(1 \mathrm{H}, \mathrm{s},=\mathrm{CH})$, $4.06\left(2 \mathrm{H}, \mathrm{q}, J\right.$ 7.1, $\left.\mathrm{OCH}_{2} \mathrm{CH}_{3}\right), 3.45\left(2 \mathrm{H}, \mathrm{t}, J\right.$ 7.1, chain $\left.\mathrm{NCH}_{2}\right), 3.26\left(2 \mathrm{H}, \mathrm{t}, J\right.$ 6.1, ring $\left.\mathrm{NCH}_{2}\right)$, 3.09 (2H, t, $J$ 6.5, $\left.\mathrm{CH}_{2} \mathrm{C}=\right), 2.54\left(2 \mathrm{H}, \mathrm{t}, J\right.$ 7.1, $\left.\mathrm{CH}_{2} \mathrm{CO}_{2} \mathrm{R}\right), 1.81-1.55$ (4H, m, ring 4-H and 5-H), $\left.1.46(9 \mathrm{H}, \mathrm{s}, \mathrm{CMe})_{3}\right)$ and $1.24\left(3 \mathrm{H}, \mathrm{t}, J \mathrm{~J} .1, \mathrm{OCH}_{2} \mathrm{CH}_{3}\right) ; \delta_{\mathrm{C}}\left(50 \mathrm{MHz} ; \mathrm{CDCl}_{3}\right) 170.9(C=\mathrm{O}$, saturated ester), $168.8\left(C=\mathrm{O}\right.$, unsaturated ester), $161.5(\mathrm{NC}=\mathrm{CH}), 82.0(\mathrm{NC}=\mathrm{CH}), 80.9\left(C \mathrm{Me}_{3}\right)$, $58.1\left(\mathrm{OCH}_{2} \mathrm{CH}_{3}\right), 50.0$ (ring $\left.\mathrm{NCH}_{2}\right)$, 47.5 (chain $\left.\mathrm{NCH}_{2}\right), 31.7\left(\mathrm{CH}_{2} \mathrm{CO}_{2} \mathrm{R}\right), 28.0\left(\mathrm{CMe}_{3}\right), 26.3$ $\left(\mathrm{CH}_{2} \mathrm{C}=\right.$ ), 23.3 (ring $\left.\mathrm{C}-5\right), 19.4$ (ring $\left.\mathrm{C}-4\right)$ and $14.6\left(\mathrm{OCH}_{2} \mathrm{CH}_{3}\right) ; \mathrm{m} / \mathrm{z}(\mathrm{EI}) 297\left(35 \%, \mathrm{M}^{+}\right), 252$ (20), 241 (20), 224 (22), 197 (17), 196 (100), 182 (40), 169 (45), 168 (40), 122 (23), 97 (45) (Found, $\mathrm{M}^{+}$, 297.1931. $\mathrm{C}_{16} \mathrm{H}_{27} \mathrm{NO}_{4}$ requires 297.1940).

Ethyl (2E)-[1-(3-hydroxypropyl)-2-pyrrolidinylidene]ethanoate (10). (a) $\mathrm{LiAlH}_{4}$ (37 mg, $0.97 \mathrm{mmol}, 1.1$ equiv.) was added to a stirred solution of tert-butyl 3-[(2E)-2-(2-ethoxy-2oxoethylidene)pyrrolidinyl]propanoate $9 \mathbf{b}(0.25 \mathrm{~g}, 0.88 \mathrm{mmol})$ in dry THF $\left(5 \mathrm{~cm}^{3}\right)$. The reaction mixture was stirred for $2 \mathrm{~h}$ at room temperature under a nitrogen atmosphere before being quenched with sequential additions of $\mathrm{H}_{2} \mathrm{O}\left(0.037 \mathrm{~cm}^{3}\right)$, aqueous $\mathrm{NaOH}$ solution $(15 \% \mathrm{w} / \mathrm{v}$, $\left.0.037 \mathrm{~cm}^{3}\right)$ and $\mathrm{H}_{2} \mathrm{O}\left(0.112 \mathrm{~cm}^{3}\right)$. A white precipitate formed. The supernatant liquid was dried $\left(\mathrm{MgSO}_{4}\right)$, filtered and evaporated in vacuo. The crude product $(0.19 \mathrm{~g})$ was purified by column chromatography on silica gel using EtOAc-hexane (4:1) as eluent to give the alcohol $\mathbf{1 0}$ as a yellow oil (0.15 g, 79\%). Recrystallisation from hexane-ethyl acetate afforded colourless needles, m.p. 62-63 ${ }^{\circ} \mathrm{C}$; $\mathrm{R}_{f} 0.42$ (EtOAc-hexane 4:1); $v_{\max }$ (film)/ $\mathrm{cm}^{-1} 3246$ (m, br, OH), 2942 (m), 1660 (s, C=O), 1586 (s, C=C), 1463 (m), 1378 (m), 1296 (m), 1253 (m), 1140 (s) and 1056 (s); $\delta_{\mathrm{H}}\left(200 \mathrm{MHz} ; \mathrm{CDCl}_{3}\right) 4.55(1 \mathrm{H}, \mathrm{br} \mathrm{s},=\mathrm{CH}), 4.07\left(2 \mathrm{H}, \mathrm{q}, J\right.$ 7.1, $\left.\mathrm{OCH}_{2} \mathrm{CH}_{3}\right), 3.65(2 \mathrm{H}, \mathrm{t}, J$ 
6.1, $\mathrm{CH}_{2} \mathrm{OH}$ ), 3.41 (2H, t, $J$ 7.1, chain $\mathrm{NCH}_{2}$ ), 3.31 (2H, t, $J$ 7.2, ring $\mathrm{NCH}_{2}$ ), 3.14 (3H, br t, $J$ 7.8, $\mathrm{CH}_{2} \mathrm{C}=$ and $\left.\mathrm{OH}\right), 1.95\left(2 \mathrm{H}\right.$, quintet, $J$ 7.5, $\left.\mathrm{CH}_{2} \mathrm{CH}_{2} \mathrm{OH}\right), 1.81$ (2H, quintet, $J$ ca. 6.7, ring 4$\mathrm{H})$ and $1.25\left(3 \mathrm{H}, \mathrm{t}, J\right.$ 7.1, $\left.\mathrm{OCH}_{2} \mathrm{CH}_{3}\right) ; \delta_{\mathrm{C}}\left(50 \mathrm{MHz}_{\mathrm{CDCl}}\right) 169.7(C=\mathrm{O}), 165.0(\mathrm{NC}=\mathrm{CH}), 77.2$ $(\mathrm{NC}=\mathrm{CH}), 59.6\left(\mathrm{CH}_{2} \mathrm{OH}\right), 58.2\left(\mathrm{OCH}_{2} \mathrm{CH}_{3}\right), 52.7$ (ring $\left.\mathrm{NCH}_{2}\right), 43.0$ (chain $\left.\mathrm{NCH}_{2}\right), 32.6$ $\left(\mathrm{CH}_{2} \mathrm{C}=\right), 28.8\left(\mathrm{CH}_{2} \mathrm{CH}_{2} \mathrm{OH}\right), 20.9$ (ring $\left.\mathrm{C}-4\right)$ and $14.6\left(\mathrm{OCH}_{2} \mathrm{CH}_{3}\right) ; \mathrm{m} / \mathrm{z}(\mathrm{EI}) 213\left(12 \%, \mathrm{M}^{+}\right)$, 169 (41), 168 (36), 126 (27), 124 (12), 122 (13), 108 (19), 98 (12), 97 (100), 96 (85) (Found: C, 61.64; H, 8.67; N, 6.99. $\mathrm{C}_{11} \mathrm{H}_{19} \mathrm{NO}_{3}$ requires C, 61.95; H, 8.98; N, 6.57\%. Found:, $\mathrm{M}^{+}, 213.1355$. $\mathrm{C}_{11} \mathrm{H}_{19} \mathrm{NO}_{3}$ requires 213.1365). (b) When the reaction was repeated with ethyl 3-[(2E)-2-(2ethoxy-2-oxoethylidene)pyrrolidinyl]propanoate 9a $(0.31 \mathrm{~g}, 1.21 \mathrm{mmol})$ and $\mathrm{LiAlH}_{4}(51 \mathrm{mg}$, $1.34 \mathrm{mmol}, 1.1$ equiv.) in dry THF $\left(5 \mathrm{~cm}^{3}\right)$, the product 10 was obtained as a yellow oil $(0.19 \mathrm{~g}$, 73\%).

Representative procedure for the reduction of ethyl 3-[(2E)-2-(2-ethoxy-2oxoethylidene)piperidinyl]propanoate (4)

To a suspension of $\mathrm{LiAlH}_{4}(21 \mathrm{mg}, 0.55 \mathrm{mmol})$ in a mixture of dry toluene and dry Et $2 \mathrm{O}(4: 1$ $\mathrm{v} / \mathrm{v}, 1 \mathrm{~cm}^{3}$ ) at $0{ }^{\circ} \mathrm{C}$ was added a solution of the vinylogous urethane 4 (144 $\mathrm{mg}, 0.54 \mathrm{mmol}$ ) in the same solvent mixture $\left(2 \mathrm{~cm}^{3}\right)$. The reaction mixture was stirred at $0{ }^{\circ} \mathrm{C}$ for 45 min before being quenched by the sequential addition of $\mathrm{H}_{2} \mathrm{O}\left(0.021 \mathrm{~cm}^{3}\right)$, aqueous $\mathrm{NaOH}$ solution $(15 \%$ $\left.\mathrm{w} / \mathrm{v}, 0.021 \mathrm{~cm}^{3}\right)$ and $\mathrm{H}_{2} \mathrm{O}\left(0.063 \mathrm{~cm}^{3}\right)$. EtOAc $\left(6.5 \mathrm{~cm}^{3}\right)$ was added, and the mixture was stirred at room temperature for $30 \mathrm{~min}$ before being filtering through celite. After evaporation of the solvent in vacuo, the residue was purified by column chromatography on silica gel using EtOAchexane (4:1) as eluent to give recovered substrate 4 (6.5 $\mathrm{mg}, 4.5 \%)$ and the following two products:

Ethyl (2E)-[1-(3-hydroxypropyl)-2-piperidinylidene]ethanoate (5). (76 mg, 62\%); pale yellow oil, $\mathrm{R}_{f} 0.24$ (EtOAc-hexane 4:1); $v_{\max }$ (film)/cm ${ }^{-1} 3419$ (s, v br, OH), 2943 (s, br), 1656 (s, C=O), 1559 (s, C=C), 1489 (m), 1448 (m), 1358 (m), 1333 (m), 1278 (m), 1140 (s, br) and 1060 (s, br); $\delta_{\mathrm{H}}\left(300 \mathrm{MHz} ; \mathrm{CDCl}_{3}\right) 4.59(1 \mathrm{H}, \mathrm{s},=\mathrm{CH}), 4.05\left(2 \mathrm{H}, \mathrm{q}, J\right.$ 7.1, $\left.\mathrm{OCH}_{2} \mathrm{CH}_{3}\right), 3.69(2 \mathrm{H}$, t, $J$ 6.0, $\mathrm{CH}_{2} \mathrm{OH}$ ), 3.31 (2H, t, $J$ 7.4, chain $\mathrm{NCH}_{2}$ ), 3.25 (2H, t, $J$ 6.1, ring $\mathrm{NCH}_{2}$ ), 3.09 (2H, t, $J$ 6.6, $\mathrm{CH}_{2} \mathrm{C}=$ ), ca. 2.6 (v br s, $\mathrm{OH}$ ), 1.85 (2H, ca. quintet, $\left.\mathrm{J} \mathrm{ca.} \mathrm{6.7,} \mathrm{CH}_{2} \mathrm{CH}_{2} \mathrm{OH}\right), 1.76(2 \mathrm{H}, c a$. quintet, $J$ ca. 6.1, ring 5-H), $1.64(2 \mathrm{H}$, ca. quintet, $J$ ca. 6.4, ring 4-H) and $1.24(3 \mathrm{H}, \mathrm{t}, J$ 7.1, $\left.\mathrm{OCH}_{2} \mathrm{CH}_{3}\right) ; \delta_{\mathrm{C}}\left(75 \mathrm{MHz} ; \mathrm{CDCl}_{3}\right) 169.1(\mathrm{C}=\mathrm{O}), 162.0(\mathrm{NC}=\mathrm{CH}), 81.1(\mathrm{NC}=\mathrm{CH}), 59.9\left(\mathrm{CH}_{2} \mathrm{OH}\right)$, $58.0\left(\mathrm{OCH}_{2} \mathrm{CH}_{3}\right)$, 49. and $48.8\left(2 \times \mathrm{NCH}_{2}\right), 28.2\left(\mathrm{CH}_{2} \mathrm{CH}_{2} \mathrm{OH}\right), 26.5\left(\mathrm{CH}_{2} \mathrm{C}=\right)$, 23.2 (ring $\left.\mathrm{C}-5\right)$, 19.4 (ring $\mathrm{C}-4)$ and $14.6\left(\mathrm{OCH}_{2} \mathrm{CH}_{3}\right) ; \mathrm{m} / \mathrm{z}(\mathrm{EI}) 227$ (13\%, $\left.\mathrm{M}^{+}\right), 196$ (27), 183 (26), 182 (72), 169 (14), 168 (11), 154 (11), 140 (100), 124 (10), 111 (30), 110 (18), 97 (28) (Found: M $^{+}, 227.1519$; $\mathrm{C}_{12} \mathrm{H}_{21} \mathrm{NO}_{3}$ requires 227.1521).

Ethyl [1-(3-hydroxypropyl)-2-piperidinyl]acetate (11). (16.5 mg, 13\%); yellow oil, $\mathrm{R}_{f} 0.13$ (EtOAc-hexane 4:1); $v_{\max }\left(\right.$ film)/cm ${ }^{-1} 3395$ (m, v br, OH), 2936 (s), 2859 (m), 1733 (s, C=O), 1446 (m), 1372 (m), 1301 (m), 1247 (m), 1162 (m, br), 1115 (m) and 1035 (m); $\delta_{\mathrm{H}}$ (200 MHz; $\left.\mathrm{CDCl}_{3}\right) 4.15\left(2 \mathrm{H}, \mathrm{q}, J\right.$ 7.1, $\left.\mathrm{OCH}_{2} \mathrm{CH}_{3}\right), 3.78\left(2 \mathrm{H}, \mathrm{t}, J\right.$ 5.2, $\left.\mathrm{CH}_{2} \mathrm{OH}\right), 3.15-3.00(1 \mathrm{H}, \mathrm{m}, \mathrm{NCH})$, 2.88-2.35 (6H, m, $2 \times \mathrm{NCH}_{2}$ and $\left.\mathrm{CH}_{2} \mathrm{CO}_{2} \mathrm{R}\right), 1.78-1.46\left(8 \mathrm{H}, \mathrm{m}\right.$, remaining $\left.\mathrm{CH}_{2}\right)$ and $1.26(3 \mathrm{H}, \mathrm{t}$, 
$J$ 7.1, $\left.\mathrm{OCH}_{2} \mathrm{CH}_{3}\right), \mathrm{OH}$ signal not located; $\delta_{\mathrm{C}}\left(50 \mathrm{MHz} ; \mathrm{CDCl}_{3}\right) 172.5(C=\mathrm{O}), 64.3\left(\mathrm{CH}_{2} \mathrm{OH}\right)$, $60.4\left(\mathrm{OCH}_{2} \mathrm{CH}_{3}\right), 57.3(\mathrm{NCH}), 54.3$ (chain $\left.\mathrm{NCH}_{2}\right), 49.2$ (ring $\left.\mathrm{NCH}_{2}\right), 34.4\left(\mathrm{CH}_{2} \mathrm{CO}_{2} \mathrm{R}\right), 30.7$ $\left(\mathrm{CH}_{2} \mathrm{CH}_{2} \mathrm{OH}\right), 27.4$ (ring $\left.\mathrm{C}-3\right), 24.9$ (ring $\left.\mathrm{C}-5\right)$ ), 21.3 (ring $\mathrm{C}-4$ ) and $14.1\left(\mathrm{OCH}_{2} \mathrm{CH}_{3}\right) ; \mathrm{m} / \mathrm{z}$ (EI) 229 (2\%, M ${ }^{+}$), 184 (21), 143 (9), 142 (100) (Found: $\mathrm{M}^{+}, 229.1674 . \mathrm{C}_{12} \mathrm{H}_{23} \mathrm{NO}_{3}$ requires 229.1678).

tert-Butyl 3-[(2E)-2-(cyanomethylene)piperidinyl]propanoate (12b). tert-Butyl 3-(2-thioxo-1piperidinyl)propanoate 8c $(0.23 \mathrm{~g}, 0.95 \mathrm{mmol})$ and bromoacetonitrile $\left(0.15 \mathrm{~cm}^{3}, 0.26 \mathrm{~g}, 2.15\right.$ mmol, 2.3 equiv.) in dry THF $\left(2 \mathrm{~cm}^{3}\right)$ were stirred at room temperature under an atmosphere of $\mathrm{N}_{2}$ for ca. $24 \mathrm{~h}$ until thin layer chromatographic analysis showed that salt formation was complete. The solvent was then evaporated in vacuo (oil pump). A solution of triethyl phosphite $\left(0.18 \mathrm{~cm}^{3}, 0.17 \mathrm{~g}, 1.05 \mathrm{mmol}\right)$ and $\mathrm{NEt}_{3}\left(0.15 \mathrm{~cm}^{3}, 0.11 \mathrm{~g}, 1.05 \mathrm{mmol}\right)$ in dry $\mathrm{CH}_{3} \mathrm{CN}\left(2 \mathrm{~cm}^{3}\right)$ was added. The mixture was stirred at room temperature for $16 \mathrm{~h}$. Dry $\mathrm{Et}_{2} \mathrm{O}$ (ca. $2 \mathrm{~cm}^{3}$ ) was added to precipitate triethylammonium bromide. The mixture was filtered through celite, and the solids were washed with $\mathrm{Et}_{2} \mathrm{O}$. The solvents were evaporated in vacuo, and the resulting orange oil was purified by column chromatography on silica gel using EtOAc-hexane (1:4) as eluent to give the product 12b as a yellow oil (0.18 g, 75\%); $\mathrm{R}_{f} 0.75$ (EtOAc-hexane 1:1); $v_{\max }$

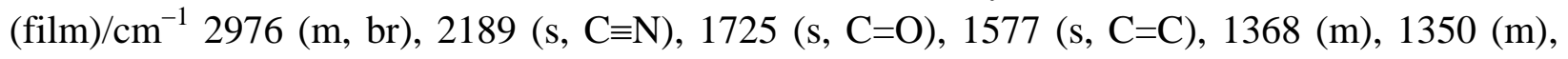
$1331(\mathrm{~m}), 1258(\mathrm{~m})$ and $1150(\mathrm{~s}, \mathrm{br}) ; \delta_{\mathrm{H}}\left(400 \mathrm{MHz} ; \mathrm{CDCl}_{3}\right) 3.60(1 \mathrm{H}, \mathrm{s},=\mathrm{CH}), 3.37$ (2H, t, J 7.1, chain $\left.\mathrm{NCH}_{2}\right), 3.22\left(2 \mathrm{H}, \mathrm{t}, J\right.$ 6.1, ring $\left.\mathrm{NCH}_{2}\right), 2.66\left(2 \mathrm{H}, \mathrm{t}, J 6.5, \mathrm{CH}_{2} \mathrm{C}=\right), 2.49(2 \mathrm{H}, \mathrm{t}, J$ 7.1, $\left.\mathrm{CH}_{2} \mathrm{CO}_{2} \mathrm{R}\right), 1.78(2 \mathrm{H}$, quintet, $J$ 6.1, ring 5-H), $1.66(2 \mathrm{H}$, quintet, $J$ 6.2, ring $4-\mathrm{H}), 1.46(9 \mathrm{H}$, s, $\left.\mathrm{CMe}_{3}\right) ; \delta_{\mathrm{C}}\left(100 \mathrm{MHz} ; \mathrm{CDCl}_{3}\right) 170.6(C=\mathrm{O}), 161.2(\mathrm{NC}=\mathrm{CH}), 122.2(\mathrm{C} \equiv \mathrm{N}), 81.2\left(\mathrm{CMe}_{3}\right), 59.6$ $(\mathrm{NC}=\mathrm{CH}), 49.8$ and $47.4\left(2 \times \mathrm{NCH}_{2}\right), 31.5\left(\mathrm{CH}_{2} \mathrm{CO}_{2} \mathrm{R}\right), 28.1\left(\mathrm{CH}_{2} \mathrm{C}=\right)$, $28.0\left(\mathrm{CMe}_{3}\right), 23.4$ (ring $\mathrm{C}-5)$ and 19.6 (ring $\mathrm{C}-4)$.

(2E)-[1-(3-Hydroxypropyl)-2-piperidinylidene]ethanenitrile (13). A solution of ethyl 3-[(2E)2-(cyanomethylene)piperidinyl]propanoate $12 \mathbf{b}(0.20 \mathrm{~g}, 0.80 \mathrm{mmol})$ in dry THF $\left(15 \mathrm{~cm}^{3}\right)$ was stirred at room temperature with $\mathrm{LiAlH}_{4}$ (33 mg, $0.88 \mathrm{mmol}, 1.1$ equiv.) for $48 \mathrm{~h}$ under an atmosphere of $\mathrm{N}_{2}$. The reaction was quenched with sequential additions of $\mathrm{H}_{2} \mathrm{O}\left(0.5 \mathrm{~cm}^{3}\right)$, aqueous $\mathrm{NaOH}$ solution $\left(15 \% \mathrm{w} / \mathrm{v}, 0.5 \mathrm{~cm}^{3}\right)$ and $\mathrm{H}_{2} \mathrm{O}\left(1.5 \mathrm{~cm}^{3}\right)$. The resulting precipitate was removed by filtration through celite, and the solids were washed with $\mathrm{CH}_{2} \mathrm{Cl}_{2}$. The combined filtrates were dried $\left(\mathrm{MgSO}_{4}\right)$, filtered and evaporated in vacuo to yield a yellow oil that was purified by column chromatography on silica gel using EtOAc-hexane (4:1) as eluent to give the alcohol 13 as a yellow oil (87 mg, 60\%); $\mathrm{R}_{f} 0.06$ (EtOAc-hexane 1:1); $v_{\max }(\mathrm{film}) / \mathrm{cm}^{-1} 3425$ (m, br, OH), 2946 (m, br), 2871 (m, br), 2184 (s, C=N), 1574 (s, C=C), 1350 (m), 1334 (m), 1176 $(\mathrm{m})$ and $1056(\mathrm{~m}) ; \delta_{\mathrm{H}}\left(400 \mathrm{MHz} ; \mathrm{CDCl}_{3}\right) 3.82(1 \mathrm{H}, \mathrm{s},=\mathrm{CH}), 3.64\left(2 \mathrm{H}, \mathrm{t}, J 5.9, \mathrm{CH}_{2} \mathrm{OH}\right), 3.25-$ $3.21\left(4 \mathrm{H}, \mathrm{m}, 2 \times \mathrm{NCH}_{2}\right)$, ca. $2.85(1 \mathrm{H}$, br s, $\mathrm{OH}), 2.65\left(2 \mathrm{H}, \mathrm{t}, \mathrm{J} 6.5, \mathrm{CH}_{2} \mathrm{C}=\right), 1.83-1.76(4 \mathrm{H}, \mathrm{m}$, $\mathrm{CH}_{2} \mathrm{CH}_{2} \mathrm{OH}$ and ring 5-H), $1.68\left(2 \mathrm{H}\right.$, quintet, $J$ 6.3, ring 4-H); $\delta_{\mathrm{C}}\left(100 \mathrm{MHz} ; \mathrm{CDCl}_{3}\right) 161.6$ $(\mathrm{NC}=\mathrm{CH}), 123.0(\mathrm{C} \equiv \mathrm{N}), 59.4\left(\mathrm{CH}_{2} \mathrm{OH}\right), 57.8(\mathrm{NC}=\mathrm{CH}), 49.5$ and $48.6\left(2 \times \mathrm{NCH}_{2}\right), 28.0$ $\left(\mathrm{CH}_{2} \mathrm{C}=\right.$ ), $27.9\left(\mathrm{CH}_{2} \mathrm{CH}_{2} \mathrm{OH}\right), 23.3$ (ring $\left.\mathrm{C}-5\right)$ and 19.4 (ring $\left.\mathrm{C}-4\right)$. Spectroscopic data were in agreement with those published previously. ${ }^{4}$ 
Ethyl 3,4,6,7,8,9-hexahydro-2H-quinolizine-1-carboxylate (6). A solution of alcohol 5 (0.48 g, $2.11 \mathrm{mmol}$ ), $\mathrm{PPh}_{3}$ (1.66 g, $6.34 \mathrm{mmol}$ ) and imidazole (0.43 g, $6.34 \mathrm{mmol}$ ) was prepared in a mixture of dry toluene and $\mathrm{CH}_{3} \mathrm{CN}\left(1: 2 \mathrm{v} / \mathrm{v}, 21 \mathrm{~cm}^{3}\right)$. Once the reactants had dissolved, iodine (1.07 g, $4.23 \mathrm{mmol})$ was added with stirring. The resulting brown solution was heated under reflux at $120^{\circ} \mathrm{C}$ for 80 min before being cooled to room temperature. The reaction mixture was washed with saturated aqueous $\mathrm{NaHCO}_{3}$ solution $\left(25 \mathrm{~cm}^{3}\right)$ and extracted with EtOAc $(2 \times$ $25 \mathrm{~cm}^{3}$ ). The combined organic phases were washed with saturated aqueous $\mathrm{NaHSO}_{3}$ solution $\left(25 \mathrm{~cm}^{3}\right)$, dried $\left(\mathrm{MgSO}_{4}\right)$, filtered and evaporated in vacuo. The crude product (2.64 g) was purified by column chromatography on silica gel using $\mathrm{CH}_{2} \mathrm{Cl}_{2}$ to elute $\mathrm{PPh}_{3}$, followed by EtOAc-hexane (1:1) to give the bicyclic product 6 as an orange oil $(0.33 \mathrm{~g}, 74 \%) ; \mathrm{R}_{f} 0.31$ (EtOAc-hexane 1:1); $v_{\max }$ (film)/cm $\mathrm{cm}^{-1} 2941$ (m), 2857 (w), 1671 (s, C=O), 1556 (s, C=C), 1320 (m), 1285 (m), 1254 (s), 1153 (m), 1118 (s) and 1065 (m); $\delta_{\mathrm{H}}\left(400 \mathrm{MHz} ; \mathrm{CDCl}_{3}\right) 4.08$ (2H, q, J 7.1, $\mathrm{OCH}_{2} \mathrm{CH}_{3}$ ), 3.11 (2H, t, $J$ 6.2, 4-H or 6-H), 3.07 (2H, t, $J$ 5.8, 4-H or 6-H), 3.01 (2H, t, $J$ 6.5, 9-H), 2.38 (2H, t, J 6.3, 2-H), 1.80-1.72 (4H, m, 3-H, 7-H), 1.61 (2H, ca. quintet, J ca. 6.3, 8-H) and $1.24\left(3 \mathrm{H}, \mathrm{t}, J\right.$ 7.1, $\left.\mathrm{OCH}_{2} \mathrm{CH}_{3}\right) ; \delta_{\mathrm{C}}\left(100 \mathrm{MHz} ; \mathrm{CDCl}_{3}\right) 168.7(\mathrm{C}=\mathrm{O}), 156.2(\mathrm{C}-9 \mathrm{a}), 91.4(\mathrm{C}-1)$, $58.2\left(\mathrm{OCH}_{2} \mathrm{CH}_{3}\right), 51.0$ (overlapping C-4, C-6), 27.6 (C-9), 23.5 (C-2), 23.2 and 21.2 (C-3, C-7), 20.4 (C-8) and $14.5\left(\mathrm{OCH}_{2} \mathrm{CH}_{3}\right) ; \mathrm{m} / \mathrm{z}$ (EI) 209 (52\%, M ${ }^{+}$), 180 (65), 178 (30), 164 (70), 162 (25), 149 (28), 137 (64), 136 (100), 135 (22), 134 (45), 122 (22), 108 (20) (Found: M+ 209.1420. $\mathrm{C}_{12} \mathrm{H}_{19} \mathrm{NO}_{2}$ requires 209.1416). Apart from the ${ }^{13} \mathrm{C}$ NMR spectrum, which has not been reported before, spectroscopic data were in agreement with those published previously. ${ }^{21}$

Ethyl (1R*,9a $\left.R^{*}\right)$-octahydro-2H-quinolizine-1-carboxylate (ethyl lupinoate) (14). A solution of ethyl 3,4,6,7,8,9-hexahydro-2H-quinolizine-1-carboxylate 6 (0.28 g, $1.34 \mathrm{mmol})$ in absolute EtOH $\left(15 \mathrm{~cm}^{3}\right)$ was stirred with Adams catalyst $(67 \mathrm{mg})$ in an autoclave under $\mathrm{H}_{2}$ gas (5 atm.) at room temperature for $16 \mathrm{~h}$. The solution was filtered through celite and washed with ethanol. The combined filtrates were evaporated in vacuo, and the crude product ( $0.28 \mathrm{~g})$ was purified by column chromatography on silica gel with EtOAc followed by MeOH-EtOAc (1:9) as eluents to give ( \pm )-ethyl lupinoate 14 as a yellow oil (0.24 g, 83\%); $\mathrm{R}_{f} 0.49$ (MeOH-EtOAc 1:4); $v_{\max }$ (film)/cm ${ }^{-1} 2935$ (s), 2857 (m), 2803 (w, Bohlmann band), 2756 (m, Bohlmann band), 2680 (w, Bohlmann band), 1738 (s, C=O), 1444 (m), 1272 (m), 1223 (m), 1207 (m), 1155 (s), 1128 (s), $1112(\mathrm{~s})$ and $1035(\mathrm{~m}) ; \delta_{\mathrm{H}}\left(400 \mathrm{MHz} ; \mathrm{CDCl}_{3}\right.$; tentative assignments by correlation experiments) 4.15-4.04 (2H, m, $\left.\mathrm{OCH}_{2} \mathrm{CH}_{3}\right), 2.90-2.85\left(2 \mathrm{H}, \mathrm{m}, 44_{\mathrm{eq}}-\mathrm{H}\right.$ and $\left.66_{\mathrm{eq}}-\mathrm{H}\right), 2.50-2.47(1 \mathrm{H}, \mathrm{m}$, $\mathrm{CHCO}_{2} \mathrm{Et}$ ), 2.20-2.05 (2H, m, 9a-H and 3-H), 2.05-1.87 (3H, m, 4ax-H, 6ax $\left.-\mathrm{H}, 8-\mathrm{H}\right), 1.72$ (1H, br d, $J$ 12.7, $\left.9_{\mathrm{ax}}-\mathrm{H}\right), 1.70-1.55(1 \mathrm{H}, \mathrm{m}, 2-\mathrm{H}), 1.55-1.45(5 \mathrm{H}, \mathrm{m}), 1.28-1.19$ and $1.21(4 \mathrm{H}$, overlapping $\mathrm{m}$ and $\mathrm{t}, J$ 7.1, $9_{\mathrm{eq}}-\mathrm{H}$ and $\left.\mathrm{OCH}_{2} \mathrm{CH}_{3}\right) ; \delta_{\mathrm{C}}\left(100 \mathrm{MHz} ; \mathrm{CDCl}_{3}\right) 173.4(C=\mathrm{O}), 62.8(\mathrm{C}-$ 9a) , $59.7\left(\mathrm{OCH}_{2} \mathrm{CH}_{3}\right), 57.2$ and 55.1 (C-4, C-6), $44.4\left(\mathrm{CHCO}_{2} \mathrm{Et}\right), 29.0(\mathrm{C}-7), 26.4(\mathrm{C}-8), 25.0$ (C-9), 24.5 (C-2), 22.1 (C-3) and $14.2\left(\mathrm{OCH}_{2} \mathrm{CH}_{3}\right) ; \mathrm{m} / \mathrm{z}$ (EI) 211 (47\%), 210 (28), 182 (72), 166 (44), 164 (22), 138 (47), 136 (37), 124 (21), 123 (21), 111 (59), 110 (47), 97 (65) (Found: M+, 211.1572. $\mathrm{C}_{12} \mathrm{H}_{21} \mathrm{NO}_{2}$ requires 211.1572). Spectroscopic data were in agreement with those reported by other workers, who did not attempt the assignment of NMR signals. ${ }^{21,22}$ 
Ethyl $\left(1 R^{*}, 9 a S^{*}\right)$-octahydro-2H-quinolizine-1-carboxylate (ethyl epilupinoate) (15). A solution of ethyl $\left(1 R^{*}, 9 \mathrm{a} R^{*}\right)$-octahydro-2H-quinolizine-1-carboxylate $14(0.34 \mathrm{~g}, 1.63 \mathrm{mmol})$ in absolute ethanol $\left(6 \mathrm{~cm}^{3}\right)$ was added dropwise to a solution of $\mathrm{NaOEt}$, prepared by dissolving a catalytic amount of $\mathrm{Na}(<5 \mathrm{mg})$ in absolute $\mathrm{EtOH}\left(6 \mathrm{~cm}^{3}\right)$. The resulting mixture was heated under reflux for $16 \mathrm{~h}$ before acidifying the solution with glacial acetic acid (30 drops). The solvent was evaporated in vacuo, and the resulting solid was extracted with $\mathrm{CH}_{2} \mathrm{Cl}_{2}\left(4 \times 30 \mathrm{~cm}^{3}\right)$. The extracts were combined and evaporated in vacuo, and the crude product $(0.70 \mathrm{~g})$ was purified by column chromatography on silica gel with MeOH-EtOAc (1:9) as eluent to give $( \pm)$ ethyl epilupinoate 15 as a pale yellow oil (0.34 g, 100\%); $\mathrm{R}_{f} 0.26$ (MeOH-EtOAc 1:4); $v_{\max }$ (film)/cm 2939 (s), 2862 (m), 2803 (w, Bohlmann band), 2755 (w, Bohlmann band), 1732 (s, $\mathrm{C}=\mathrm{O}), 1446$ (m), 1373 (m), 1317 (m), 1260 (s), 1175 (s), 1146 (s), $1034(\mathrm{~m})$ and $733(\mathrm{~m}) ; \delta_{\mathrm{H}}$ $\left(200 \mathrm{MHz} ; \mathrm{CDCl}_{3}\right) 4.14$ (2H, q, J 7.2, $\left.\mathrm{OCH}_{2} \mathrm{CH}_{3}\right), 3.09$ (2H, br t, $J$ ca 10.7, $4_{\mathrm{eq}}-\mathrm{H}$ and 6 eq $\left.-\mathrm{H}\right)$, 2.57-2.49 (1H, m, CHCO $2 \mathrm{Et}), 2.34-2.19$ (3H, m), 1.98-1.96 (1H, m), 1.85-1.65 (6H, m), 1.61$1.29(3 \mathrm{H}, \mathrm{m})$ and $1.25\left(3 \mathrm{H}, \mathrm{t}, J\right.$ 7.1, $\left.\mathrm{OCH}_{2} \mathrm{CH}_{3}\right) ; \delta_{\mathrm{C}}\left(50 \mathrm{MHz} ; \mathrm{CDCl}_{3}\right) 174.1(C=\mathrm{O}), 63.5(\mathrm{C}-9 \mathrm{a})$, $60.4\left(\mathrm{OCH}_{2} \mathrm{CH}_{3}\right), 55.9$ and 55.2 (C-4 and C-6), $47.8\left(\mathrm{CHCO}_{2} \mathrm{Et}\right), 29.5$ (C-7), 28.2 (C-8), 24.4 (C9), $23.6(\mathrm{C}-2), 23.3(\mathrm{C}-3)$ and $14.1\left(\mathrm{OCH}_{2} \mathrm{CH}_{3}\right)$. Spectroscopic data were in agreement with those reported by other workers. ${ }^{21,22}$

$\left(1 R^{*}, 9 \mathrm{a} R^{*}\right)$-Octahydro-2H-quinolizin-1-ylmethanol, ( \pm )-lupinine (1). A solution of ethyl $\left(1 R^{*}, 9 \mathrm{a} R^{*}\right)$-octahydro-2H-quinolizine-1-carboxylate $14(0.11 \mathrm{~g}, 0.51 \mathrm{mmol})$ in dry $\mathrm{Et}_{2} \mathrm{O}\left(2 \mathrm{~cm}^{3}\right)$ was added to a stirred suspension of $\mathrm{LiAlH}_{4}$ (29 mg, $0.76 \mathrm{mmol}, 1.5$ equiv.) in dry $\mathrm{Et}_{2} \mathrm{O}\left(1 \mathrm{~cm}^{3}\right)$ cooled to $0{ }^{\circ} \mathrm{C}$. After $1 \mathrm{~h}$, the reaction was quenched by sequential additions of $\mathrm{H}_{2} \mathrm{O}\left(0.029 \mathrm{~cm}^{3}\right)$, aqueous $\mathrm{NaOH}$ solution $\left(15 \% \mathrm{w} / \mathrm{v}, 0.029 \mathrm{~cm}^{3}\right)$ and $\mathrm{H}_{2} \mathrm{O}\left(0.087 \mathrm{~cm}^{3}\right)$. A white precipitate formed. EtOAc $\left(6 \mathrm{~cm}^{3}\right)$ was added, and after a further $30 \mathrm{~min}$ of stirring, the mixture was filtered through celite. The filtrate was evaporated in vacuo, and the crude product $(0.10 \mathrm{~g})$ was purified by column chromatography on silica gel with $\mathrm{MeOH}-\mathrm{EtOAc}(1: 4)$ as eluent to give $( \pm$ )-lupinine $\mathbf{1}$ as a pale yellow oil (87 mg, 95\%); $\mathrm{R}_{f} 0.33$ (MeOH-EtOAc 1:4); $v_{\max }(\mathrm{film}) / \mathrm{cm}^{-1} 3333$ (m, v br, $\mathrm{OH}$ ), 2934 (s), 2857 (m), 2806 (m, Bohlmann band), 2762 (m, Bohlmann band), 2677 (w, Bohlmann band), 1444 (m), 1351 (m), 1114 (m), 1088 (m), 1066 (m) and 1037 (s); $\delta_{\mathrm{H}}$ (300 $\mathrm{MHz}$; $\mathrm{C}_{6} \mathrm{D}_{6}$; assignments follow ref. 25) $4.76(1 \mathrm{H}$, br s, $\mathrm{OH}), 4.14(1 \mathrm{H}, \mathrm{dd}, J 10.7$ and 6.1, $\left.\mathrm{CH}_{\mathrm{a}} \mathrm{H}_{\mathrm{b}} \mathrm{OH}\right), 3.75\left(1 \mathrm{H}\right.$, dd, $J 10.7$ and 2.0, $\left.\mathrm{CH}_{\mathrm{a}} \mathrm{H}_{\mathrm{b}} \mathrm{OH}\right), 2.56-2.46\left(2 \mathrm{H}, \mathrm{m}, 4_{\mathrm{eq}}-\mathrm{H}, 6_{\mathrm{eq}}-\mathrm{H}\right), 2.31-$ $2.17\left(1 \mathrm{H}, \mathrm{m}, 3_{\mathrm{ax}}-\mathrm{H}\right), 1.80-1.68(3 \mathrm{H}, \mathrm{m}), 1.62(1 \mathrm{H}, \mathrm{dd}, J 12.0$ and 3.4, $4 \mathrm{ax}-\mathrm{H}), 1.60-1.50(2 \mathrm{H}, \mathrm{m})$, 1.45-1.15 (6H, m) and $1.00\left(1 \mathrm{H}\right.$, qt, $J 12.5$ and 3.8, 8ax-H); $\delta_{\mathrm{C}}\left(75 \mathrm{MHz} ; \mathrm{C}_{6} \mathrm{D}_{6}\right) 65.2(\mathrm{C}-9 \mathrm{a}$ and $\mathrm{CH}_{2} \mathrm{OH}$ ), 57.3 (C-4 and C-6), 39.1 (C-1), 31.2 (C-2), 29.9 (C-9), 25.9 (C-7), 25.0 (C-8) and 23.1 (C-3); $\delta_{\mathrm{C}}\left(100 \mathrm{MHz} ; \mathrm{CDCl}_{3}\right) 65.5\left(\mathrm{CH}_{2} \mathrm{OH}\right), 64.9$ (C-9a), 56.9 (C-4 and C-6), 38.2 (C-1), 30.9 (C-2), 29.4 (C-9), 25.4 (C-7), 24.5 (C-8) and 22.7 (C-3). Spectroscopic data were in agreement with those reported by other workers. ${ }^{22,25}$

$\left(1 R^{*}, 9 a S^{*}\right)-O c t a h y d r o-2 H$-quinolizin-1-ylmethanol, ( \pm )-epilupinine (2). A solution of ethyl $\left(1 R^{*}, 9 \mathrm{a} S^{*}\right)$-octahydro-2H-quinolizine-1-carboxylate $15(0.29 \mathrm{~g}, 1.39 \mathrm{mmol})$ in dry $\mathrm{Et}_{2} \mathrm{O}\left(6 \mathrm{~cm}^{3}\right)$ was added to a stirred suspension of $\mathrm{LiAlH}_{4}$ (63 mg, $1.67 \mathrm{mmol}, 1.2$ equiv.) in dry $\mathrm{Et}_{2} \mathrm{O}\left(3 \mathrm{~cm}^{3}\right)$ cooled to $0{ }^{\circ} \mathrm{C}$. After $75 \mathrm{~min}$, the reaction mixture was filtered through celite, and solids were 
and washed with undried (moist) $\mathrm{Et}_{2} \mathrm{O}$. The filtrate was evaporated in vacuo to yield epilupinine 2 as a chromatographically pure pale yellow oil $(0.22 \mathrm{~g}, 88 \%)$ that decomposed if purification on silica gel was attempted; $\mathrm{R}_{f} 0.15$ (ca. 1\% aq. $\mathrm{NH}_{3}$ in EtOAc); $v_{\max }$ (film)/ $/ \mathrm{cm}^{-1} 3342$ and 3207 (m, v br, OH), 2930 (s), 2858 (m), 2807 (m, Bohlmann band), 2760 (m, Bohlmann band), 2678 (w, Bohlmann band), 1674 (m), 1443 (m), 1371 (m), 1296 (m), 1113 (m), 1093 (m), 1070 (m) and $1014(\mathrm{w}) ; \delta_{\mathrm{H}}\left(300 \mathrm{MHz} ; \mathrm{CDCl}_{3}\right) 3.65\left(1 \mathrm{H}\right.$, dd, $J 10.8$ and 3.6, $\left.\mathrm{CH}_{\mathrm{a}} \mathrm{H}_{\mathrm{b}} \mathrm{OH}\right), 3.51(1 \mathrm{H}, \mathrm{dd}, J 10.8$ and 6.0, $\left.\mathrm{CH}_{\mathrm{a}} \mathrm{H}_{\mathrm{b}} \mathrm{OH}\right), 3.28(1 \mathrm{H}, \mathrm{br} \mathrm{s}, \mathrm{OH}), 2.80\left(2 \mathrm{H}, \mathrm{br} \mathrm{t}, J 13.2,4_{\mathrm{eq}}-\mathrm{H}\right.$ and $\left.6_{\mathrm{eq}}-\mathrm{H}\right), 2.04-1.98(2 \mathrm{H}$, m, 4 ax $-\mathrm{H}$ and $\left.66_{\mathrm{ax}}-\mathrm{H}\right), 1.93-1.84(2 \mathrm{H}, \mathrm{m}), 1.79-1.57(6 \mathrm{H}, \mathrm{m}), 1.45-1.36\left(1 \mathrm{H}, \mathrm{m}, \mathrm{CHCH}_{2} \mathrm{OH}\right)$ and 1.23-1.16 (3H, m); $\delta_{\mathrm{C}}\left(75 \mathrm{MHz} ; \mathrm{CDCl}_{3}\right) 64.3(\mathrm{C}-9 \mathrm{a}), 64.0\left(\mathrm{CH}_{2} \mathrm{OH}\right), 56.7$ and $56.5(\mathrm{C}-4$ and C6), 43.8 (C-1), 29.5 and, 28.1 (C-2 and C-9), 25.4 (C-7), 24.8 (C-8) and 24.4 (C-3). Spectroscopic data were in agreement with those reported by other workers. ${ }^{22}$

\section{Acknowledgements}

We thank the National Research Foundation, Pretoria and the University of the Witwatersrand for providing the funding for this research and for bursaries to CSF. We are indebted to Miss C. M. Jungmann and Dr C. A. Meerholz for carrying out some preliminary experiments, Dr A. S. Howard for invaluable discussions, and Dr P. R. Boshoff (Cape Technikon) and Mrs S. Heiss (University of the Witwatersrand) for recording mass spectra and NMR spectra, respectively.

\section{References}

1. Michael, J. P.; de Koning, C. B.; Gravestock, D.; Hosken, G. D.; Howard, A. S.; Jungmann, C. M.; Krause, R. W. M.; Parsons, A. S.; Pelly, S. C.; Stanbury, T. V. Pure Appl. Chem. 1999, 71, 979.

2. For representative examples, see the following: (a) Pyrrolidines: Michael, J.P.; Parsons, A. S. Tetrahedron 1996, 52, 2199. (b) Perhydroindoles: Michael, J. P.; Howard, A. S.; Katz, R. B.; Zwane, M. I. Tetrahedron Lett. 1992, 33, 6023. (c) Pyrrolo[1,2-a]quinolines: Michael, J. P.; de Koning, C. B.; Hosken, G. D.; Stanbury, T. V. Tetrahedron 2001, 57, 9635. (d) Pyrrolo[1,2-a]indoles: Michael, J. P.; de Koning, C. B.; Petersen, R. L.; Stanbury, T. V. Tetrahedron Lett. 2001, 42, 7513.

3. (a) Michael, J. P.; Gravestock, D. S. Afr. J. Chem. 1998, 51, 146. (b) Michael, J. P.; Gravestock, D. J. Chem. Soc., Perkin Trans. 1 2000, 1919 and references cited therein.

4. Michael, J. P.; Jungmann, C. M. Tetrahedron 1992, 48, 10211.

5. Michael, J. P.; de Koning, C. B.; van der Westhuyzen, C. W.; Fernandes, M. A. J. Chem. Soc., Perkin Trans. 1 2001, 2055.

6. (a) Michael, J. P. In The Alkaloids. Chemistry and Biology, Cordell, G. A. Ed.; Academic Press: New York, 2001; Vol. 55, pp 91-258. (b) Howard, A. S.; Michael, J. P. In The 
Alkaloids. Chemistry and Pharmacology, Brossi, A. Ed.; Academic Press: New York, 1986; Vol. 28, pp 183-308.

7. For annual reports on progress in the chemistry of indolizidine and quinolizidine alkaloids, see Michael, J. P. Nat. Prod. Rep. 2001, 18, 520 and earlier reviews in this series.

8. (a) Ohmiya, S.; Saito, K.; Murakoshi, I. In The Alkaloids. Chemistry and Pharmacology, Cordell, G. A. Ed.; Academic Press: San Diego, 1995; Vol. 47, pp 1-114. (b) Aslanov, K. A.; Kushmuradov, Y. K.; Sadykov, A. S. In The Alkaloids. Chemistry and Pharmacology, Brossi, A. Ed.; Academic Press: New York, 1987; Vol. 31, pp 117-192.

9. For syntheses of lupinine and epilupinine reported between 1985 and 1992, see ref. 8(a); for those reported between 1992 and 1999, see ref. 1. For more recent syntheses, see the following: (a) Padwa, A.; Heidelbaugh, T. M.; Kuethe, J. T. J. Org. Chem. 2000, 65, 2368. (b) Ledoux, S.; Marchalant, E.; Célérier, J.-P.; Lhommet, G. Tetrahedron Lett. 2001, 42, 5397. (c) Huang, H.-L.; Sung, W.-H.; Liu, R.-S. J. Org. Chem. 2001, 66, 6193. (d) Tate, E. W.; Zard, S. Z. Tetrahedron Lett. 2002, 43, 4683.

10. Gerrans, G. C.; Howard, A. S.; Orlek, B. S. Tetrahedron Lett. 1975, 4171.

11. Goldberg, S. I.; Ragade, I. J. Org. Chem. 1967, 32, 1046.

12. Brillon, D. Synth. Commun. 1990, 20, 3085.

13. Halasa, A. F.; Smith C. E. P. J. Org. Chem. 1971, 36, 636.

14. (a) Roth, M.; Dubs, P.; Götschi, E.; Eschenmoser, A. Helv. Chim. Acta 1971, 54, 710. (b) Shiosaki, K. In Comprehensive Organic Synthesis, Trost, B. M., Ed.; Pergamon Press: Oxford, 1991; Vol. 2, pp 865-892.

15. Davidson, R. S.; Günther, W. H. H.; Waddington-Feather, S. M.; Lythgoe, B. J. Chem. Soc. 1964, 4907.

16. Fisher, G. B.; Harrison, J.; Fuller, J. C.; Goralski, C.T.; B. Singaram, B. Tetrahedron Lett 1992, 33, 4533.

17. Sudharshan M.; Hultin, P. G. Synlett 1997, 171.

18. (a) Brown, H. C.; Brewster, J. H.; Shechter, H. J. Am. Chem. Soc. 1954, 76, 467. (b) Brown, H. C. J. Org. Chem. 1957, 22, 439.

19. Garegg, P. J.; Samuelson, B. J. Chem. Soc., Perkin Trans. 1 1980, 2866.

20. Goldberg, S. I.; Lipkin, A. H. J. Org. Chem. 1970, 35, 242.

21. (a) Haddad, M.; Célérier, J.-P.; Lhommet, G. Heterocycles 1987, 26, 2335. (b) Célérier, J.P.; Haddad, M.; Saliou, C.; Lhommet, G.; Dhimane, H.; Pommelet, J. C.; Chuche, J. Tetrahedron 1989, 45, 6161.

22. Hua, D. H.; Miao, S. W.; Bravo, A. A.; Takemoto, D. J. Synthesis 1991, 970.

23. (a) Bohlmann, F.; Schumann, D.; Schulz, M. Tetrahedron Lett. 1964, 173. (b) Bohlmann, F. Chem. Ber. 1958, 91, 2157.

24. Davies, S. G.; Smyth, G. D. J. Chem. Soc., Perkin Trans 1 1996, 2467.

25. Rycroft, D. S.; Robins, D. J. Magn. Reson. Chem. 1992, 30, S15.

26. Daly, J. W.; Garraffo, H. M.; Spande, T. F. In Alkaloids: Chemical and Biological Perspectives, Pelletier, S. W. Ed.; Pergamon Press: Amsterdam, 1999; Vol. 13, pp 1-161. 
27. Howard, A. S.; Gerrans, G. C.; Meerholz, C. A. Tetrahedron Lett. 1980, 21, 1373. 